PROCEEDINGS OF THE

AMERICAN MATHEMATICAL SOCIETY

Volume 125, Number 1, January 1997, Pages 251-254

S 0002-9939(97)03535-1

\title{
COVERING BY COMPLEMENTS OF SUBSPACES, II
}

\author{
W. EDWIN CLARK AND BORIS SHEKHTMAN
}

(Communicated by Jeffry N. Kahn)

\begin{abstract}
Let $V$ be an $n$-dimensional vector space over an algebraically closed field $K$. Define $\gamma(k, n, K)$ to be the least positive integer $t$ for which there exists a family $E_{1}, E_{2}, \ldots, E_{t}$ of $k$-dimensional subspaces of $V$ such that every $(n-k)$-dimensional subspace $F$ of $V$ has at least one complement among the $E_{i}$ 's. Using algebraic geometry we prove that $\gamma(k, n, K)=k(n-k)+1$.
\end{abstract}

\section{INTRODUCTION}

Take $V=V(n, K)$ to be an $n$-dimensional vector space over the algebraically closed field $K$. As usual a subspace $F$ of $V$ is a complement of the subspace $E$ of $V$ if $V=E \oplus F$, i.e., if $E+F=V$ and $E \cap F=\{0\}$. We let $c(E)$ denote the set of all complements of $E$ in $V$ and we write $G(k, n)$ for the set of all $k$-subspaces ( $=k$-dimensional subspaces) of $V$. If $E \in G(k, n)$ then $c(E) \subseteq$ $G(n-k, n)$. Define $\gamma(k, n, F)$ to be the least positive integer $t$ such that there exist $k$-subspaces $E_{1}, E_{2}, \ldots, E_{t}$ of $V$ satisfying

$$
c\left(E_{1}\right) \cup c\left(E_{2}\right) \cup \cdots \cup c\left(E_{t}\right)=G(n-k, n) .
$$

If (1) holds we say that all $(n-k)$-subspaces of $V$ are covered by the $E_{i}$ 's.

In [1] we studied this problem for an arbitrary field $K$. Among other things we showed that in general $\gamma(k, n, K)$ depends on the field K. In particular, we showed that $\gamma(2,4, K)$ is 5 if $K$ is quadratically closed and is 4 otherwise. We conjectured that $\gamma(k, n, K)=k(n-k)+1$ if $K$ is algebraically closed. Here we prove this conjecture using results from algebraic geometry.

\section{The LOWER Bound $k(n-k)+1 \leq \gamma(k, n, K)$}

Let $\Lambda^{k}(V)$ denote the $k$-vectors in the exterior algebra $\Lambda(V)$ of $V$. We let $D(k, n)$ denote the set of all non-zero decomposable $k$-vectors $\alpha=v_{1} \wedge v_{2} \wedge \cdots \wedge v_{k}$ where $v_{1}, v_{2}, \ldots, v_{k}$ are linearly independent vectors in $V$. Let $\langle\alpha\rangle$ denote the 1dimensional subspace of $\Lambda^{k}(V)$ generated by $\alpha$ and write

$$
\overline{D(k, n)}=\{\langle\alpha\rangle \mid \alpha \in D(k, n)\} .
$$

If $v_{1}, v_{2}, \ldots, v_{k}$ is a basis for $E \in G(k, n)$, then the mapping $E \mapsto\left\langle v_{1} \wedge \cdots \wedge v_{k}\right\rangle$ is a bijection from $G(k, n)$ to $\overline{D(k, n)}$. It is well-known that this gives $G(k, n)$ the

Received by the editors November 22, 1994 and, in revised form, July 6, 1995.

1991 Mathematics Subject Classification. Primary 51A99; Secondary 14N10, 15A75, 15 A99.

Key words and phrases. Vector space, subspace, complement, projective variety, Grassmannian. 
structure of an irreducible projective variety (the Grassmannian ) of dimension $k(n-k)$ in $\mathbb{P}^{N}=\mathbb{P}\left(\Lambda^{k}(V)\right)$ where $N=\left(\begin{array}{l}n \\ k\end{array}\right)-1$. We identify $G(k, n)$ with $\overline{D(k, n)}$.

Now for any positive integer $t$ let $G(k, n)^{t}$ be the product variety of $G(k, n)$ with itself $t$ times. Let $E=\left(E_{1}, \ldots, E_{t}\right) \in G(k, n)^{t}$. For each $i$ let $E_{i}=\left\langle\epsilon_{i}\right\rangle$ for some decomposable $\epsilon_{i} \in \Lambda^{k}(V)$. Define the mappings:

$$
\varphi_{i}: \Lambda^{n-k}(V) \rightarrow \Lambda^{n}(V) \quad \text { by } \quad \varphi_{i}(\xi)=\epsilon_{i} \wedge \xi
$$

for $i=1, \ldots, t$ and let

$$
\mathcal{K}(E)=\operatorname{ker}\left(\varphi_{1}\right) \cap \operatorname{ker}\left(\varphi_{2}\right) \cap \cdots \cap \operatorname{ker}\left(\varphi_{t}\right) .
$$

Note that $\mathcal{K}(E)$ is a subspace of $\Lambda^{n-k}(V)$.

Lemma 1. For $E \in G(k, n)^{t}$ the following two conditions are equivalent:

(a) $c\left(E_{1}\right) \cup c\left(E_{2}\right) \cup \cdots \cup c\left(E_{t}\right)=G(n-k, n)$,

(b) $D(n-k, n) \cap \mathcal{K}(E)=\emptyset$.

Proof. This is an immediate consequence of the fact that if $F=\langle\alpha\rangle \in G(n-k, n)$ for some $\alpha \in D(n-k, n)$, then $E_{i} \cap F=\{0\}$ if and only if $\epsilon_{i} \wedge \alpha \neq 0$.

Lemma 2. If $\gamma(k, n, K)=t$ and $E=\left(E_{1}, \ldots, E_{t}\right) \in G(k, n)^{t}$ satisfies

$$
c\left(E_{1}\right) \cup c\left(E_{2}\right) \cup \cdots \cup c\left(E_{t}\right)=G(n-k, n),
$$

then

$$
\operatorname{dim}(\mathcal{K}(E))=\left(\begin{array}{l}
n \\
k
\end{array}\right)-t
$$

Proof. Since $\varphi_{i}$ is a linear mapping from the $\left(\begin{array}{l}n \\ k\end{array}\right)$-dimensional vector space $\Lambda^{n-k}(V)$ to the 1-dimensional vector space $\Lambda^{n}(V)$, it suffices to show that the mappings

$$
\varphi_{i} \in \operatorname{hom}\left(\Lambda^{n-k}(V), \Lambda^{n}(V)\right), \quad i \in\{1, \ldots, t\},
$$

are linearly independent. To see this we first note that the elements $\epsilon_{i}$ are linearly independent in $\Lambda^{n-k}(V)$. Suppose not; then we can assume that $\epsilon_{t}=\sum_{i=1}^{t-1} a_{i} \epsilon_{i}$. It follows that $\bigcap_{i=1}^{t} \operatorname{ker}\left(\varphi_{i}\right)=\bigcap_{i=1}^{t-1} \operatorname{ker}\left(\varphi_{i}\right)$. This implies by Lemma 1 that

$$
c\left(E_{1}\right) \cup c\left(E_{2}\right) \cup \cdots \cup c\left(E_{t-1}\right)=G(n-k, n)
$$

and hence $\gamma(k, n, K) \leq t-1$, a contradiction. Now assume that the mappings $\varphi_{1}, \ldots, \varphi_{t}$ are linearly dependent. Say, $\sum_{i=1}^{t} a_{i} \varphi_{i}=0$. This means that for all $\xi \in \Lambda^{n-k}(V)$ we have $0=\sum_{i=1}^{t} a_{i}\left(\epsilon_{i} \wedge \xi\right)=\left(\sum_{i=1}^{t} a_{i} \epsilon_{i}\right) \wedge \xi$. So it suffices to observe that if $\delta \in \Lambda^{k}(V)$ and if $\delta \wedge \xi=0$ for all $\xi \in \Lambda^{n-k}(V)$ then $\delta=0$.

Lemma 3. If $K$ is any algebraically closed field, then

$$
k(n-k)+1 \leq \gamma(k, n, K) .
$$

Proof. Suppose $\gamma(k, n, K)=t \leq k(n-k)$. Then there exists $E=\left(E_{1}, \ldots, E_{t}\right) \in$ $G(k, n)^{t}$ such that $c\left(E_{1}\right) \cup \cdots \cup c\left(E_{t}\right)=G(n-k, n)$. So by Lemmas 1 and 2 there is a linear subspace $\mathcal{K}(E)$ of $\Lambda^{n-k}(V)$ such that $D(k, n) \cap \mathcal{K}(E)=\emptyset$ and $\mathcal{K}(E)$ has affine dimension $\left(\begin{array}{l}n \\ k\end{array}\right)-t$ which is at least $\left(\begin{array}{l}n \\ k\end{array}\right)-k(n-k)$. Let $\mathcal{K}^{\prime}$ denote the 
corresponding projective subspace of $\mathbb{P}\left(\Lambda^{n-k}(V)\right)$. Then $\mathcal{K}^{\prime} \cap G(n-k, n)=\emptyset$. But using projective dimensions we have [3, Proposition 11.4]

$$
\begin{aligned}
\operatorname{dim}\left(\mathcal{K}^{\prime}\right)+\operatorname{dim}(G(n-k, n)) & \geq\left(\begin{array}{l}
n \\
k
\end{array}\right)-k(n-k)-1+k(n-k) \\
& \geq\left(\begin{array}{l}
n \\
k
\end{array}\right)-1=\operatorname{dim}\left(\mathbb{P}\left(\Lambda^{n-k}(V)\right)\right)
\end{aligned}
$$

and it follows that $\mathcal{K}^{\prime} \cap G(n-k, n) \neq \emptyset$ which is a contradiction.

3. The UPPER Bound $\gamma(k, n, K) \leq k(n-k)+1$

Lemma 4. If $K$ is algebraically closed, then

$$
\gamma(k, n, K) \leq k(n-k)+1 .
$$

Proof. Let $\nu=k(n-k)$ denote the dimension of $G(k, n)$ (and $G(n-k, n)$ ) as a projective variety. Let

$$
A=G(k, n)^{\nu+1} .
$$

Then $A$ is a projective variety of dimension $\nu(\nu+1)$. For every $F \in G(n-k, n)$ define

$$
B(F)=\{E \in G(k, n) \mid E \cap F \neq 0\} .
$$

Now $\mathrm{B}(\mathrm{F})$ is an irreducible projective variety with

$$
\operatorname{dim}(B(F))=\nu-1 .
$$

For $F \in G(n-k, n)$ define

$$
C(F)=B(F)^{\nu+1} \text {. }
$$

Then

$$
\operatorname{dim}(C(F))=(\nu+1)(\nu-1)=\nu^{2}-1 .
$$

Now set

$$
C=\bigcup_{F \in G(n-k, n)} C(F) .
$$

Note that if $C$ is properly contained in $A$, then there exists $E=\left(E_{1}, \ldots, E_{\nu+1}\right) \in$ $A-C$. Then for all $F \in G(n-k, n)$ we have $E \notin C(F)$ so there must exist an index $i \in\{1, \ldots, \nu+1\}$ such that $E_{i} \cap F=0$. Hence $c\left(E_{1}\right) \cup \cdots \cup c\left(E_{\nu+1}\right)=G(n-k, n)$ and so $\gamma(k, n, K) \leq \nu+1$, as desired. So it remains only to show that $C$ is properly contained in $A$. In fact we claim that $C$ is a variety of dimension at most $\operatorname{dim}(A)-1=\nu^{2}+\nu-1$.

To complete the proof we fix $F_{0} \in G(n-k, n)$ and consider the projective variety

$$
D:=C\left(F_{0}\right) \times P G L_{n}(K) .
$$

We note that

$$
\operatorname{dim}(D)=\operatorname{dim}\left(C\left(F_{0}\right)\right)+\operatorname{dim}(P G L(n, K))=\nu^{2}-1+n^{2}-1 .
$$

An element $M$ of $P G L(n, K)$ induces a linear automorphism of $\mathbb{P}\left(\Lambda^{k}(V)\right)$ which induces in turn an automorphism of $G(k, n)$. Abusing notation we write $U \mapsto M U$ 
to indicate the latter automorphism. Now we define $\varphi: D \rightarrow C$ as follows: For $(E, M) \in D$ set

$$
\varphi(E, M)=\left(M E_{1}, M E_{2}, \ldots, M E_{\nu+1}\right) .
$$

Clearly $\varphi$ is a regular surjection. Hence by [3, Theorem 11.12]

$$
\operatorname{dim}(D)=\operatorname{dim}(C)+\mu
$$

where

$$
\mu=\min \left\{\operatorname{dim}\left(\varphi^{-1}\left(E^{\prime}\right)\right\}, \quad E^{\prime} \in C .\right.
$$

This shows that

$$
\operatorname{dim}(C)=\nu^{2}-1+n^{2}-1-\mu .
$$

So to prove that $\operatorname{dim}(C) \leq \nu^{2}+\nu-1$ it suffices to prove that $n^{2}-\nu-1 \leq \mu$. To see this consider the subset $G(F)$ of $P G L_{n}(K)$ whose elements map the fixed $(n-k)$ subspace $F_{0}$ to the $(n-k)$-space $F$. It is easy to see that $\operatorname{dim}(G(F))=n^{2}-\nu-1$. Now if $E^{\prime}=\left(E_{1}^{\prime}, \ldots, E_{\nu+1}^{\prime}\right) \in C(F) \subseteq C$ then for each $M \in G(F)$ we have

$$
\left(M^{-1} E^{\prime}, M\right)=\left(M^{-1} E_{1}^{\prime}, \ldots, M^{-1} E_{\nu+1}^{\prime}, M\right) \in \varphi^{-1}\left(E^{\prime}\right) .
$$

The mapping $M \mapsto\left(M^{-1} E^{\prime}, M\right)$ is a regular injection from $G(F)$ into the fiber $\varphi^{-1}\left(E^{\prime}\right)$. It follows that each fiber has dimension at least that of $G(F)$ and this completes the proof.

Remarks. 1. The above proof shows that almost all $\left(E_{1}, \ldots, E_{\nu+1}\right) \in G(k, n)^{\nu+1}$ satisfy

$$
c\left(E_{1}\right) \cup c\left(E_{2}\right) \cup \cdots \cup c\left(E_{\nu+1}\right)=G(n-k, n)
$$

since the complement $C$ of the set of such $(\nu+1)$-tuples forms a variety of dimension smaller than $\operatorname{dim}\left(G(k, n)^{\nu+1}\right)$

2. As shown in [1] $\gamma(2,4, K)=4$ when $K$ is not quadratically closed. So the lower bound $\gamma(k, n, K) \geq k(n-k)+1$ proved here for algebraically closed fields will not hold in general. On the other hand, we suspect that the upper bound $\gamma(k, n, K) \leq k(n-k)+1$ does hold for arbitrary fields. In fact we have verified this for finite fields of sufficiently large order using counting arguments [2]. However, as the referee pointed out it is slightly worrying that the conjecture fails in the "thin" case, that is, if we replace $n$-space by $n$-set, $k$-subspace by $k$-subset and vector space complement by set complement. However, the upper bound of $\left(\begin{array}{l}n \\ k\end{array}\right)$ given in [1] holds in both cases.

\section{REFERENCES}

1. W. Edwin Clark and Boris Shekhtman, Covering by complements of subspaces, Linear and Multilinear Algebra 40 (1995), 1-13. CMP 96:08

2. _ Domination numbers of q-analogues of Kneser graphs, Bulletin of the Institute of Combinatorics and its Applications (to appear).

3. J. Harris, Algebraic Geometry, Springer-Verlag, 1992. MR 93j:14001

Department of Mathematics, University of South Florida, Tampa, Florida 33620-5700

E-mail address: eclark@math.usf.edu

E-mail address: boris@math.usf.edu 